Relations industrielles

Industrial Relations

\title{
La CTCC et l'affillation au CTC
}

\section{Gérard Dion}

Volume 13, numéro 1, janvier 1958

URI : https://id.erudit.org/iderudit/1022466ar

DOI : https://doi.org/10.7202/1022466ar

Aller au sommaire du numéro

Éditeur(s)

Département des relations industrielles de l'Université Laval

ISSN

0034-379X (imprimé)

1703-8138 (numérique)

Découvrir la revue

Citer ce document

Dion, G. (1958). La CTCC et l'affillation au CTC. Relations industrielles / Industrial Relations, 13(1), 57-61. https://doi.org/10.7202/1022466ar

Tous droits réservés (C Département des relations industrielles de l’Université Laval, 1958
Ce document est protégé par la loi sur le droit d'auteur. L’utilisation des services d'Érudit (y compris la reproduction) est assujettie à sa politique d'utilisation que vous pouvez consulter en ligne.

https://apropos.erudit.org/fr/usagers/politique-dutilisation/ 
duelle, mais de considérer l'ensemble du mouvement ouvrier. En dépit de certains articles de journaux, trop spéculatifs à notre avis, notre mouvement doit sortir de cette conférence plus uni, plus déterminé, plus militant que jamais.

Vous avez la responsabilité de décider de l'avenir de plusieurs milliers d'ouvriers dans nos unions locales. Cette responsabilité dépasse nos opinions personnelles, dépasse les préjugés que nous pourrions avoir. Je sais que vous vous montrerez à la hauteur de ces graves responsabilités. Que ces trois jours puissent marquer d'une pierre blanche l'histoire du mouvement ouvrier de cette province.

\section{LA CTCC ET L'AFFILIATION AU CTC}

\section{GÉrard Dion}

La question des rapports à établir entre la CTCC et le CTC intéresse à juste titre tout le public canadien. Nous avons déjà retracé dans le numéro spécial de Relations Industrielles sur la fusion CTMC-CCT les attitudes officielles prises par la CTCC en ces dernières années. ${ }^{1}$

Comme le congrès de 1956 s'était prononcé en faveur du principe de l'affiliation de la CTCC au CTC et qu'une attitude définitive devrait être prise au cours d'un congrès spécial qui aurait lieu au plus tard le ler juin 1957, on s'attendait que le congrès annuel de 1957 apporterait quelque chose de nouveau. Or il n'en n'a été à peu près rien.

Tout d'abord le congrès spécial a été décommandé parce qu'une question préalable n'avait pu être réglée: celle de la réforme des structures internes de la CTCC.

La question a cependant rubondi devant le congrès. Deux résolutions venant de groupes particuliers s'opposaient à l'affiliation. L'une d'elle, celle de la Fédération du bâtiment (numéro 68) ne donnait aucun considérant. L'autre, celle de la Fédération des employés des corporations municipales et scolaires (numéro 69), se présentait comme suit:

«Considérant que l'affiliation (ou la fusion) est un problème complexe; Considérant que le Comité de l'Unité syndicale n'a pas terminé son étude; Considérant que la C.T.C.C. n'a pas soumis de mémoire à l'Episcopat; Considérant que l'unité ouvrière au Canada ne sera possible que le jour où les locaux affiliés au Congrès du Travail du Canada se libéreront de leur affiliation aux unions américaines pour constituer un mouvement authentiquement canadien tenant compte des réalités politiques canadiennes;

Considérant que l'unité qui existe au sein du nouveau Congrès ou du Congrès unifié semble précaire étant donné que les unions vivent dans un état d'absolue indépendance les unes des autres et à l'égard de la centrale même;

Il est recommandé:

a) de prier la CTCC de soumettre à la Commission Episcopale d'Action Sociale le mémoire demandé et de ne prendre aucune décision relativement à la fusion ou à l'affiliation sans avoir au préalable reçu l'avis de l'Episcopat sur ce sujet.

b) de s'opposer à tout projet d'affiliation ou de fusion au Congrès du travail du Canada tant et aussi longtemps que les conditions énumérées ci-dessus n'auront pas été remplies. »

(1) «La CTCC et l'unité syndicale canadienne», par Gérard Dion, daus Relations Industrielles, vol. 12, no 1-2, pp. 32-55. 
En somme, cette fédération s'objectait à l'affiliation pour deux raisons fondamentales à la nature et la structure du CTC et elle alléguait deux prétextes pour que le congrès ne prenne aucune attitude en ce moment: la question n'a pas été soumise à l'épiscopat et le comité de l'unité syndicale n'a pas terminé son étude.

Or le Comité de l'unité syndicale recommanda le rejet de la proposition dans les termes suivants:

«Le Congrès de 1956 s'est prononcé en faveur du principe de l'affiliation de la C.T.C.C. au C.T.C. Avant que cette décision ne soit prise, les officiers supérieurs de la C.T.C.C. avaient pressenti des membres éminents du Corps Episcopal canadien à ce sujet.

Le sermon de Son Eminence le Cardinal Léger à l'ouverture de notre Congrès, l'an dernier, et l'allocution de notre aumônier général, faite au cours de ce même congrès, ont réglé, dans l'esprit des membres du Comité, la question du principe de l'affiliation pourvu que nous prenions les mesures de prudence nécessaires à la protection de l'intégrité du mouvement. C'est donc dans les conditions d'affiliation que nous pourrons juger de la valeur de ces garanties: il faut distinguer entre une mesure qui viserait à modifier l'orientation doctrinale du mouvement et une autre qui ne fait qu'établir un lien entre la C.T.C.C. et une centrale nationale. »

Le Comité avait préparé un projet déterminant les conditions d'affiliation de la CTCC au CTC selon les décisions prises au congrès de l'an dernier .Il le présenta donc en recommandant son adoption. Voici le texte du projet:

Le Congrès du Travail du Canada (CTC) et la Confédération des Travailleurs Catholiques du Canada (CTCC) conviennent que l'unité syndicale au Canada, établie sur des bases mutuellement acceptables, peut mieux assurer la promotion des intérêts et la réalisation des aspirations légitimes des travailleurs canadiens de même qu'elle permettra de mieux servir la cause de la paix, de la liberté et de la sécurité dans le monde.

En vue d'atteindre cet objectif de l'unité syndicale au Canada, le Congrès du Travail du Canada (CTC) a adopté, à sa Convention historique de fondation, en avril 1956, à Toronto, les deux résolutions suivantes:

\section{1.-RESOLU:}

Que cette Convention historique de fondation du Congrès du Travail du Canada confie à son nouvel Exécutif le soin de négocier, avec toutes les autres organisations syndicales démocratiques et bona fide, les conditions qui permettraient de les accepter, le plus rapidement possible, dans ce grand Congrès .

\section{2.-RESOLU:}

Que le Congrès du Travail du Canada accueille favorablement les propositions de la Confédération des Travailleurs Catholiques du Canada en vue de faire progresser l'unité syndicale canadienne;

Et qu'il soit également résolu que le nouvel Exécutif du CTC étudie les conditions de l'affiliation de la Confédération des Travailleurs Catholiques du Canada au Congrès du Travail du Canada.

La C.T.C.C. ayant adopté, à sa convention de septembre 1956, à Montréal, le principe de son affiliation au Congrès du Travail du Canada, les comités d'unité syndicale formés par les deux organisations en vue d'examiner cette question ont arrêté et s'engagent à recommander les conditions suivantes d'affiliation de la C.T.C.C. au C.T.C.

1) La Confédération des Travailleurs Catholiques du Canada demande officiellement son affiliation au Congrès du Travail du Canada.

2) La C.T.C.C. reconnaît que sa première obligation, dans l'éventualité d'une affiliation, est de se conformer à la constitution du Congrès du Travail 
du Canada, à la politique et aux règlements qui en découlent, en tenant compte, toutefois, des dispositions de la présente entente.

3) A compter de la date de son affiliation, la C.T.C.C. aura le statut d'une union nationale affiliée au Congrès du Travail du Canada Il sest convenu que tous les organismes affiliés à la C.T.C.C. font partie intégrante de cette union nationale.

4) Pour éviter toute confusion, la C.T.C.C. choisira un nom plus approprié à son nouveau statut.

5) La C.T.C.C. n'admet pas qu'on applique un traitement injuste à cause de la langue, de la nationalité, de la race, du sexe et de la religion . Elle tiendra compte de ce principe dans les modifications constitutionnelles qu'elle opérera à l'occasion de son affiliation au Congrès du Travail du Canada.

6) Dans les limites ci-haut décrites, la C.T.C.C. conserve son entière autonomie en tout ce qui a trait à son orientation doctrinale et à ses structures internes. et la présente entente ne peut être interprétée comme modifiant ses caractéristiques propres ou portant atteinte à son intégrité.

7) A la suite de son affiliation au CTC, la CTCC cessera d'agir comme centrale syndicale sur le plan canadien et abandonnera son affiliation à la Confédération Internationale des Syndicats Chrétiens (CISC). Quant à ses représentants sur les Conseils et Commissions relevant de la juridiction fédérale (Ottawa), ils resteront en fonction, en qualité de représentants du Congrès du Travail du Canada, jusqu'à la fin de leur terme d'office ou jusqu'à ce que le Congrès leur ait désigné des successeurs.

8) Les Conseils Centraux affiliés à la C.T.C.C. devront, dans un délai d'au plus deux ans, se conformer aux dispositions de la Constitution du Congrès du Travail du Canada concernant les Conseils du Travail. Toutefois, si à cause des services qu'il donne, l'un de nos conseils continuait d'exister avec l'accord des syndicats intéressés, soit sous un même nom, soit sous la forme d'un conseil conjoint (joint Board) ou d'un autre organisme de ce genre, cette solution sera admise par le CTC.

9) La CTCC abandonnera, dans un délai d'au plus deux ans, ses fonctions de représentation législative dans la province de Québec et recommandera à ses organismes de s'affilier à la Fédération des Travailleurs du Québec (CTC).

10) Comme toute autre union nationale affiliés au CTC, la CTCC pourra tenir des Congrès, conformément à sa constitution, mais les résolutions adoptées seront transmises au Congrès du Travail du Canada, s'il s'agit de sujets relevant de la juridiction fédérale, et à la Fédération des Travailleurs du Québec, s'il s'agit de sujets de juridiction provinciale.

11) Tout conflit de juridiction entre la CTCC et une autre organisation affiliée au CTC sera réglé en la manière prévue dans la Constitution du Congrès du Travail du Canada.

12) Lors de la prochaine Convention du Congrès du Travail du Canada, le Conseil Exécutif du CTC s'engage à recommander un quatrième vice-président pour la Province de Québec.

13) Le Congrès du Travail du Canada continuera de publier dans les deux langues officielles sa Constitution, ses Règlements, sa Revue mensuelle et ses rapports et bulletins aux organisations affiliées. De plus, lors des Conventions régulières ou spéciales, un service de traduction simultanée des délibérations dans les deux langues officielles sera mis à la disposition de tous les délégués.

14) Les droits et privilèges reconnus à la CTCC dans la présente entente ne peuvent être modifiés que sur accord des parties.

Ce projet donna lieu à une longue discussion ainsi qu'à un amendement et à un sous-amendement.

Nous tenons à reproduire textuellement l'amendement, car il reflète la pensée d'un fort groupe de délégués. 
«La CTCC demande son affiliation au Congrès du Travail du Canada: «Considérant que, dans les conditions actuelles, une forte partie des locaux qui constituent le Congrès du Travail du Canada est formée de locaux d'unions internationales ou américaines, cette demande d'affiliation soit cependant conditionnée de la façon suivante:

1. La CTCC gardera son statut de centrale syndicale, nationale et provinciale;

2. La CTCC gardera son affiliation à la Confédération Internationale des Syndicats Chrétiens (CISC);

3. La CTCC restera maîtresse exclusive de son orientation doctrinale, de sa liberté d'expansion et de toutes ses structures internes.

Il était évident qu'un tel amendement qui posait des conditions si évidemment inadmissibles par le CTC équivalait purement et simplement au rejet d'une affiliation future.

C'est alors que le sous-amendement suivant a été adopté avec 204 congressistes votant en sa faveur et 189 contre.

1.-La Confédération des Travailleurs Catholiques du Canada demande officiellement son affiliation au Congrès du Travail du Canada.

2.-La CTCC reconnaît que sa première obligation, dans l'éventualité d'une affiliation, est de se conformer à la constitution du Congrès du Travail du Canada, à la politique et aux règlements qui en découlent, en tenant compte, toutefois, des dispositions de la présente entente.

3.-A compter de la date de son affiliation, la CTCC aura le statut d'une union nationale affiliée au Congrès du Travail du Canada. Il est convenu que tous les organismes affiliés à la CTCC font partie intégrante de cette union nationale.

4.--Pour éviter toute confusion, la CTCC choisira un nom plus approprié à son nouveau statut.

5.-La CTCC n'admet pas qu'on applique un traitement injuste à cause de la langue, de la nationalité, de la race, du sexe et de la religion. Elle tiendra compte de ce principe dans les modifications constitutionnelles qu'elle opérera à l'occasion de son affiliation au Congrès du Travail du Canada.

6) La CTCC conserve son entière autonomie en tout ce qui a trait à son orientation doctrinale, à son expansion et à ses structures internes et, la présente entente ne peut être interprétée comme modifiant ses caractéristiques propres ou portant atteinte à son intégrité.

7.-A la suite de son affiliation au CTC, la CTCC cessera d'agir comme centrale syndicale sur le plan canadien et réexaminera l'opportunité de maintenir son affiliation à la Confédération Internatioale des Syndicats Chrétiens (CISC). Quant à ses représentants sur les Conseils et Commissions relevant de la juridiction fédérale (Ottawa), ils resteront en fonction, en qualité de représentants du Congrès du Travail du Canada, jusqu'à la fin de leur terme d'office ou jusquà ce que le Congrès leur ait désigné des successeurs. 8.-La CTCC pourra tenir des Congrès conformément à sa constitution, mais les résolutions adoptées seront transmises au Congrès du Travail du Canada, s'il s'agit de sujets relevant de la juridiction fédérale.

9.-Lors de la prochaine Convention du Congrès du Travail du Canada, le Conseil Exécutif du CTC s'engage à recommander un quatrième vice-président pour la Province de Québec.

10.-Le Congrès du Travail du Canada continuera de publier dans les deux langues officielles sa Constitution, ses Règlements, sa Revue mensuelle et ses rapports et bulletins aux organisations affiliées. De plus, lors des Conventions régulières ou spéciales, un service de traduction simultanée des délibérations dans les deux langues officielles sera mis à la disposition de tous les délégués. 11.-Les droits et privilèges reconnus à la CTCC dans la présente entente ne peuvent être modifiés que sur accord des parties. » 
La position officielle de la CTCC se trouve donc exprimée dans cette résolution. Pour en comprendre toute la portée, il faut se référer à la proposition même du comité de l'unité syndicale qui était explicite, peut-être trop explicite aux yeux de plusieurs congressistes. Les points marquants des différences sont les suivants:

a) Le Congrès de la CTCC n'est pas prêt à ce que les Conseils centraux cessent d'exister et de jouer un rôle.

b) La CTCC se réserve le droit d'une action législative sur le plan provincial et en s'engage pas pour le moment à s'affilier à la Fédération des Travailleurs du Québec.

c) La CTCC conserve son autonomie en ce qui a trait à son expansion.

d) La CTCC, au lieu d'abandonner immédiatement son affiliation à la CISC, s'engage seulement à en réexaminer l'opportunité.

Il faut dire que ce sont aussi les points les plus litigieux dans les négociations entre la CTCC et le CTC et qu'ils peuvent être facilement des pierres d'achoppement à l'affiliation. Tous n'ont pas la même importance.

Aussi, il serait contradictoire que la CTCC, cessant d'exister comme centrale nationale, continue d'être affiliée à la CISC qui est actuellement formée des centrales nationales. Mais que la constitution de la CISC soit modifrée et permette l'affiliation de tous les groupements particuliers qui s'inspirent de la doctrine sociale chrétienne, il n'y aura plus de problème.

De plus, que la CTCC continue de présenter des revendications législatives au nom de ses membres exclusivement, il aurait valu mieux ne pas le mentionner, car c'est déjà une pratique courante. De même, soulever le problème des juridictions pour l'expansion de la CTCC, c'est une question théoriquement insoluble que seul le temps et l'expérience neuvent régler. Ici, il aurait valu mieux s'entendre sur un code d'éthique professionnelle entre groupements.

Le texte de la décision du Congrès de la CTCC lie ses représentants dans leurs pourparlers avec ceux du CTC et toute modification devra recevoir l'approbation d'un nouveau congrès avant d'être agréé. De plus, la faible majorité avec laquelle cette décision a été prise laisse clairement entrevoir que, dans la CTCC, l'ensemble des groupements ne sont pas prêts à accepter facilement l'affiliation au CTC. Mais il reste qu'en prenant cette position le Congrès de la CTCC n'a pas complètement fermé toutes les portes à une affiliation éventuelle et que les négociations peuvent se continuer. Peut-être qu'avec le temps et surtout des changements d'attitudes sur le plan des locaux et des syndicats, les oppositions farouches viendront à s'atténuer. On distinguera plus clairement alors entre l'essentiel et l'accessoire, et on trouvera le moyen de concilier les tendances diverses en sauvegardant tout ce qui mérite d'être conservé pour le bien de la classe ouvrière. Il ne faut pas s'illusionner, ni aller trop vite, car cela ne peut se faire du jour au lendemain.

\section{N'OU B LIEZ PAS}

\section{Le Congrès des Relations industrielles de Laval \\ les 21 et 22 arril 1958 \\ au Château Frontenac}

